Original paper

\title{
Evaluation of different diagnostic modalities of minimal hepatic encephalopathy in cirrhotic patients: case-control study
}

\author{
Tary Salman', Maha Elsabaawy', Mahmoud Omar ${ }^{1}$, Mohamed Afify ${ }^{1}$, Hossam Elezawy', Samar Ghanem¹, \\ Osama Abdelraouf', Eman Rewisha', Nashwa Shebl' ${ }^{1}$ \\ ${ }^{1}$ National Liver Institute, Menoufia University, Egypt \\ 2Faculty of Computers and Information, Menoufia University, Egypt
}

\begin{abstract}
Introduction: Minimal hepatic encephalopathy (MHE) represents one of the most overlooked complications of liver cirrhosis.

Aim of the study: To compare the utility and efficacy of different MHE diagnostic modalities.

Material and methods: This case-control study was conducted on hepatitis C virus (HCV)-related compensated cirrhotic patients. The Psychometric Hepatic Encephalopathy Score (PHES) was used to assign patients to MHE and controls. All patients were subjected to plasma ammonia, serum 3-nitrotyrosine (3-NT), critical flicker frequency (CFF), and the modified inhibitory control test (ICT).

Results: CFF was significantly lower in the control group $(38.5,40 \mathrm{~Hz}, p=0.003)$. The unweighted lures on ICT were 8.7, 4.9 in MHE and controls $(p<0.001)$. Moreover, ammonia was higher in the MHE group $(89,61.5 \mu \mathrm{mol} / \mathrm{l}, p<0.001)$. 3-NT was also higher in the MHE group $(31.5,13.7 \mathrm{nmol} / \mathrm{l}, p<0.001)$ respectively. CFF at cutoff $<39 \mathrm{~Hz}$ had sensitivity, specificity, positive predictive value (PPV) and negative predictive value (NPV) of $57.5 \%, 77.5 \%, 71.9 \%$ and $64.6 \%$, respectively; in modified ICT, at cutoff $>5$ unweighted lures the values were $87.5 \%, 80 \%, 81.4 \%$ and $86.5 \%$, respectively; in ammonia, at cutoff $\geqslant 76.45 \mu \mathrm{mol} / \mathrm{l}$ the values were $65 \%, 72.5 \%, 70.3 \%$ and $67.4 \%$, respectively; for $3-\mathrm{NT}$ at cutoff $\geqslant 14.15 \mathrm{nmol} / \mathrm{l}$ the values were $85 \%$, $82.5 \%, 82.9 \%$ and $84.6 \%$, respectively. The accuracy for MHE diagnosis was $67.5 \%, 83.3 \%, 68.8 \%, 83.8 \%$ relying on CFF, 3-NT, ammonia, and ICT respectively. On multivariate analysis, $\mathrm{CFF}<39 \mathrm{~Hz}(\mathrm{OR}=10.2, p=0.04)$, modified ICT $>5$ unweighted lures ( $O R=43.2, p=0.002)$, and serum 3-NT levels $\geqslant 14.15 \mathrm{nmol} / \mathrm{I}(\mathrm{OR}=50.4$, $p<0.001$ ) were independent predictors of MHE.
\end{abstract}

Conclusions: 3-NT and ICT are advantageous to reveal MHE in compensated liver cirrhosis, while CFF can be only used as adjuncts, with humble merits of ammonia.

Key words: minimal hepatic encephalopathy, psychometric tests, psychometric hepatic encephalopathy score, critical flicker frequency, inhibitory control test, serum 3-nitrotyrosine levels, liver cirrhosis, ammonia, diagnosis, overt.

Address for correspondence:

Prof. Maha Elsabaawy, National Liver Institute, Menoufia University, Egypt, e-mail: maha.ahmed@liver.menofia.edu.eg

\section{Introduction}

Hepatic encephalopathy (HE) is a brain dysfunction caused by liver insufficiency and/or portosystemic shunting; it manifests as a wide spectrum of neurological or psychiatric abnormalities ranging from subclinical alterations to coma [1]. It represents a spectrum of reversible cognitive changes ranging from mild inattention and executive function deficits to more advanced stages marked by lethargy, disorientation, and even coma $[2,3]$. Although the pathogenesis of HE is multifactorial [4], ammonia remains the best-characterized neurotoxin implicated in the pathogenesis of HE [5]. 
The International Society for Hepatic Encephalopathy and Nitrogen Metabolism (ISHEN) consensus outlined the symptoms and their grading into covert (minimal HE [MHE] and grade I) and overt HE (OHE, grades II-IV) in September 2014 [1]. MHE is highly prevalent albeit underrecognized - among patients with cirrhosis, affecting up to $80 \%$ of this subset of patients [6]. It negatively affects quality of life for patients and their families [7]. MHE has been shown to interfere with daily functioning, such as the ability to safely operate a vehicle [8]. The diagnosis of MHE can be challenging. Patients with MHE have normal findings on clinical examination, but abnormal psychometric test results. Subtle symptoms of MHE can only be diagnosed through specialized neuropsychiatric testing [9].

The gold standard for MHE is formal consultation with an expert neuropsychologist to administer and score a battery of timed paper-pencil tests in a controlled setting [10]. This is costly, often unavailable, and therefore most clinicians do not routinely employ such testing for their patients [11]. In the past decade, point-of-care alternatives have emerged. These include the critical flicker frequency $(\mathrm{CFF})$ test $[12,13]$, the inhibitory control test (ICT) [14], and the Stroop test, which has been integrated into a smartphone app (EncephalApp) [15], among others. These tests have not been adequately validated in a real-world setting and a consensus on the corresponding optical cutoff points has not been reached. In a previous study, serum levels of 3-nitrotyrosine (were shown to be a good predictor of the presence of MHE in patients with cirrhosis [16]. Our aim was to evaluate the utility and accuracy of CFF, a modified version of ICT and serum 3-nitrotyrosine levels in the diagnosis of MHE, compared to the gold standard - Psychometric Hepatic Encephalopathy Score (PHES).

\section{Material and methods}

This case control study was conducted on 80 patients with hepatitis $\mathrm{C}$ virus (HCV)-related compensated liver cirrhosis who presented to the outpatient clinics at National Liver Institute, Menoufia University in Egypt for their regular follow-up visits. HCV was documented by positive PCR HCV RNA. Cirrhosis was confirmed by the clinical, laboratory, and radiological characteristics [1]. Absence of decompensation is established by absence of variceal bleeding, ascites, jaundice, and hepatic encephalopathy [1].

The control group was selected from age- and sexmatched healthy volunteers.

Inclusion criteria of the study were patients with compensated liver cirrhosis aged 18-70, with no previous episodes of OHE. HBV-related chronic liver disease, or with known neurological effects such as recent or old infarction, Wilson disease, hepatocellular carcinoma, alcohol abuse, drug abuse and/or the concurrent use of neuropsychiatric drugs constituted the exclusion criteria of the study. Illiterate patients were also excluded.

\section{Study protocol}

Approval of the National Liver Institute's Ethical Committee, along with written informed consent, were obtained from the enrolled subjects prior to commencement in the study. Demographic data were collected for the study subjects. Thorough history taking and physical examination - including pelviabdominal ultrasound - were then conducted. Patients underwent baseline laboratory testing, including liver function tests, renal function tests and complete blood counts. Positive HCV PCR RNA, along with negative viral markers for hepatitis $B$ virus (HBV) infection, was determined. Furthermore, Child-Pugh score and FIB-4 index were calculated for the study subjects. All patients were subsequently subjected, on the same day each, to psychometric testing, the CFF test, ICT and measurements of plasma ammonia and serum 3-nitrotyrosine levels.

\section{Psychometric Hepatic Encephalopathy Score}

The Psychometric Hepatic Encephalopathy Score (PHES) - a battery that includes five paper-pencil tests - was used as the gold standard to define MHE and subsequently assign patients to one of the two study groups (MHE and control groups). PHES included number connection tests (NCTs) $\mathrm{A}$ and $\mathrm{B}$, the line drawing test, the serial dotting test and a digit symbol test. Performance on each test was scored in seconds and expressed as standard deviations ( $Z$-scores) from the average values obtained from healthy controls [10]. The PHES was the sum of each component: -3 for $Z<-3$, -2 for $Z<-2,-1$ for $Z<-1,0$ for $-1<Z<1,1$ for $Z>1$. PHES was considered abnormal with a $Z$-score cutoff $\leq-4$ [17-19], with subsequent assignment of the patient to the MHE group.

\section{Critical flicker frequency test}

Critical flicker frequency was defined as the frequency at which a fused light (presented from $60 \mathrm{~Hz}$ downward) appeared to be flickering to the observer. Studies have shown its reduction with worsening cognition and improvement after therapy $[12,13]$.

\section{Modified inhibitory control test}

Inhibitory control test is a computerized test of response inhibition and working memory that is freely 
Table 1. Classification of cases according to PHES

\begin{tabular}{ll}
\hline PHES & \\
\hline Normal (>-4 SD) & $40(50 \%)$ \\
\hline Impaired & \\
\hline-4 SD & $27(33.75 \%)$ \\
\hline-6 SD & $13(16.25 \%)$ \\
\hline
\end{tabular}

MHE - minimal hepatic encephalopathy, PHES - psychometric hepatic encephalopathy score

downloadable at www.hecme.tv [14]. While the original version of the test used the English letters $\mathrm{X} / \mathrm{Y}$ to define targets of the test, we adapted a special modification to the test by using the numbers $7 / 8$ in Arabic $(v / \wedge)$ as targets; this modification was created by expert staff members at the Faculty of Computers and Information, Menoufia University, to suit Egyptian patients.

The plasma ammonia test procedure was done using a Cobas Integra 800 Auto analyzer (Roche Diagnostics Ltd., Germany, catalogue number: M, 87432).

Serum 3-nitrotyrosine levels: it was measured using the quantitative sandwich enzyme immunoassay technique (ELISA). Adding the substrate to a complex of 3-nitrotyrosine antigen, antibody, and a catalyzing enzyme; makes it blue. This is followed by adding the HRP catalyzing enzyme. The reaction is terminated by the addition of a sulfuric acid solution and the color change is measured spectrophotometrically at a wavelength of $450 \mathrm{~nm}$. The concentration of 3-nitrotyrosine in the samples is then determined by comparing the O.D. of the samples to the standard curve.

\section{Statistical analyses}

Data were analyzed using SPSS statistical software (version 24, IBM Corp., Armonk, NY, USA). Numerical variables were expressed as means and standard deviation (SD). Categorical variables were expressed as numbers and percentages. The independent $t$-test was used to compare means for numerical variables between the study groups. The $\chi^{2}$ test was used to compare categorical variables. Receiver operating characteristic (ROC) curves were drawn to estimate the accuracy of different tests and measures in the diagnosis of MHE. The area under the ROC (AUROC) curve was then calculated. An optimal cutoff value with the most acceptable accuracy was selected for each test and/or measure. A $p$-value was considered significant below 0.05 .

\section{Results}

Forty patients (50\%) scored higher than -4 SD on PHES and comprised our control group. The remaining forty patients (50\%), who scored $-4 \mathrm{SD}$ or lower on PHES, comprised the MHE group (Table 1).

The mean age of the two study groups were 50.65 $\pm 6.91,52.88 \pm 6.7$ years in the control and MHE groups respectively. Forty-nine patients $(61.3 \%)$ were female. There were no significant differences between the two study groups regarding age, gender, aspartate aminotransferase (AST), alanine aminotransferase (ALT), serum albumin, serum bilirubin levels, platelet count, Child-Pugh score and FIB-4 index (Table 2).

Overall, patients scored $39.3 \pm 2.3 \mathrm{~Hz}$ on the CFF test and $6.78 \pm 3.43$ unweighted lures on modified ICT. Plas-

Table 2. Differences in the baseline characteristics and demographics between the study groups

\begin{tabular}{|c|c|c|c|c|}
\hline Variable & $\begin{array}{l}\text { Control group } \\
(n=40)\end{array}$ & $\begin{array}{l}\text { MHE group } \\
(n=40)\end{array}$ & $\chi^{2}$ & $p$-value \\
\hline \multicolumn{5}{|l|}{ Gender } \\
\hline Male & $18(58.1 \%)$ & $13(41.9 \%)$ & 1.317 & 0.251 \\
\hline \multirow[t]{2}{*}{ Female } & $22(44.9 \%)$ & 27 (55.1\%) & & \\
\hline & \multicolumn{2}{|c|}{ Mean $\pm S D$} & $t$-test & $p$-value \\
\hline Age (years) & $50.65 \pm 6.91$ & $52.88 \pm 6.7$ & -1.462 & 0.148 \\
\hline AST (U/I) & $43.73 \pm 23.55$ & $40.18 \pm 18.68$ & 0.747 & 0.457 \\
\hline $\mathrm{ALT}(\mathrm{U} / \mathrm{l})$ & $35.38 \pm 19.33$ & $33.83 \pm 16.99$ & 0.381 & 0.704 \\
\hline Serum albumin $(\mathrm{g} / \mathrm{dl})$ & $3.87 \pm 0.34$ & $3.81 \pm 0.33$ & 0.731 & 0.467 \\
\hline Serum bilirubin (mg/dl) & $0.83 \pm 0.29$ & $0.77 \pm 0.26$ & 0.984 & 0.328 \\
\hline Platelet count $\left(\times 10^{3} / \mathrm{mm}^{3}\right)$ & $122.32 \pm 47.38$ & $108.93 \pm 38.98$ & 1.381 & 0.171 \\
\hline Child-Pugh score & $5.23 \pm 0.62$ & $5.13 \pm 0.34$ & 0.898 & 0.372 \\
\hline FIB-4 index & $3.35 \pm 1.37$ & $3.69 \pm 1.82$ & -0.947 & 0.346 \\
\hline
\end{tabular}

MHE - minimal hepatic encephalopathy 
Table 3. Tests of minimal hepatic encephalopathy in the study groups

\begin{tabular}{|c|c|c|c|c|}
\hline \multirow[t]{2}{*}{ Variable } & $\begin{array}{l}\text { Control group } \\
(n=40)\end{array}$ & $\begin{array}{l}\text { MHE group } \\
(n=40)\end{array}$ & \multirow[t]{2}{*}{$t$-test } & \multirow[t]{2}{*}{$p$-value } \\
\hline & \multicolumn{2}{|c|}{ Mean \pm SD } & & \\
\hline Critical flicker frequency (Hz) & $40.02 \pm 1.73$ & $38.53 \pm 2.47$ & 3.122 & $0.003^{*}$ \\
\hline $\begin{array}{l}\text { Modified ICT } \\
\text { (unweighted lures) }\end{array}$ & $4.9 \pm 2.47$ & $8.65 \pm 3.24$ & -5.824 & $<0.001^{*}$ \\
\hline Plasma ammonia ( $\mu \mathrm{mol} / \mathrm{l})$ & $61.52 \pm 21.41$ & $88.99 \pm 36.81$ & -4.081 & $<0.001^{*}$ \\
\hline Serum 3-nitrotyrosine (nmol/l) & $13.69 \pm 4.2$ & $31.48 \pm 22.35$ & -4.949 & $<0.001^{*}$ \\
\hline
\end{tabular}

Table 4. Area under the receiver operating characteristic curve (AUROC) of different measures and tests of minimal hepatic encephalopathy in the study population

\begin{tabular}{lccc}
\hline Variable & AUROC & $95 \% \mathrm{CI}$ & $p$-value \\
\hline Critical flicker frequency $(\mathrm{Hz})$ & 0.670 & $0.551-0.789$ & $0.009^{*}$ \\
\hline Modified ICT (unweighted lures) & 0.827 & $0.732-0.921$ & $<0.001^{*}$ \\
\hline Plasma ammonia $(\mu \mathrm{mol} / \mathrm{l})$ & 0.735 & $0.623-0.847$ & $<0.001^{*}$ \\
\hline Serum 3-nitrotyrosine $(\mathrm{nmol} / \mathrm{l})$ & 0.854 & $0.763-0.945$ & $<0.001^{*}$ \\
\hline
\end{tabular}

Cl - confidence interval, ICT - inhibitory control test, * significant p-value

ma ammonia and serum 3-nitrotyrosine levels were 75.3 $\pm 33 \mu \mathrm{mol} / \mathrm{l}$ and $22.6 \pm 18.3 \mathrm{nmol} / \mathrm{l}$ in the study subjects, respectively (Table 3 ).

\section{Univariate analysis of different measures and tests of minimal hepatic encephalopathy in the study groups}

Patients in the MHE group scored significantly lower on the CFF test, compared to the control group $(38.5 \mathrm{~Hz}$ vs. $40 \mathrm{~Hz}, p=0.003)$. The mean number of unweighted lures on modified ICT was 8.7 and 4.9 in the MHE and control groups, respectively $(p<0.001)$. Moreover, plasma ammonia levels were significantly higher in the MHE group, compared to the control group (89 vs. $61.5 \mu \mathrm{mol} / \mathrm{l}$, $p<0.001)$. Serum 3-nitrotyrosine levels were also significantly higher in the MHE group, compared to the control group (31.5 vs. $13.7 \mathrm{nmol} / \mathrm{l}, p<0.001)$. The results of the univariate analyses are tabulated in Table 4.

\section{Accuracy of different measures and tests of minimal hepatic encephalopathy in the study groups}

ROC curves were drawn for different predictors and tests of MHE (Fig. 1). AUROC was then estimated. An optimal cutoff point with the most acceptable accuracy was selected for each test and measure (Table 5).

CFF test: AUROC was 0.670 (95\% CI: 0.551-0.789, $p=0.009)$. A cutoff of $<39 \mathrm{~Hz}$ had a sensitivity, speci- ficity, positive predictive value (PPV) and negative predictive value (NPV) of $57.5 \%, 77.5 \%, 71.9 \%$ and $64.6 \%$, respectively with an overall accuracy of $67.5 \%$ in the diagnosis of MHE in the study population (Fig. 1A, Table 5).

Modified ICT: AUROC was 0.827 (95\% CI: 0.732$0.921, p<0.001)$. A cutoff of $>5$ unweighted lures had a sensitivity, specificity, PPV and NPV of $87.5 \%, 80.0 \%$, $81.4 \%$ and $86.5 \%$, respectively with an overall accuracy of $83.8 \%$ in the diagnosis of MHE in the study population (Fig. 1B, Table 5).

Plasma ammonia: AUROC was 0.735 (95\% CI: $0.623-0.847, p<0.001)$. A cutoff of $\geq 76.45 \mu \mathrm{mol} / 1 \mathrm{had}$ a sensitivity, specificity, PPV and NPV of $65.0 \%, 72.5 \%$, $70.3 \%$ and $67.4 \%$, respectively with an overall accuracy of $68.8 \%$ in the diagnosis of MHE in the study population (Fig. 1C, Table 5).

Serum 3-nitrotyrosine: AUROC was 0.854 (95\% CI: $0.763-0.945, p<0.001)$. A cutoff of $\geq 14.15 \mathrm{nmol} / 1 \mathrm{had}$ a sensitivity, specificity, PPV and NPV of $85.0 \%, 82.5 \%$, $82.9 \%$ and $84.6 \%$, respectively with an overall accuracy of $83.8 \%$ in the diagnosis of MHE in the study population (Fig. 1D, Table 5).

\section{Multivariate analysis of predictors of minimal hepatic encephalopathy in the study population}

On multivariate analysis, CFF test $<39 \mathrm{~Hz}$ $(\mathrm{OR}=10.2, p=0.04)$, modified ICT $>5$ unweighted lures $(\mathrm{OR}=43.2, p=0.002)$, and serum 3-nitrotyrosine 

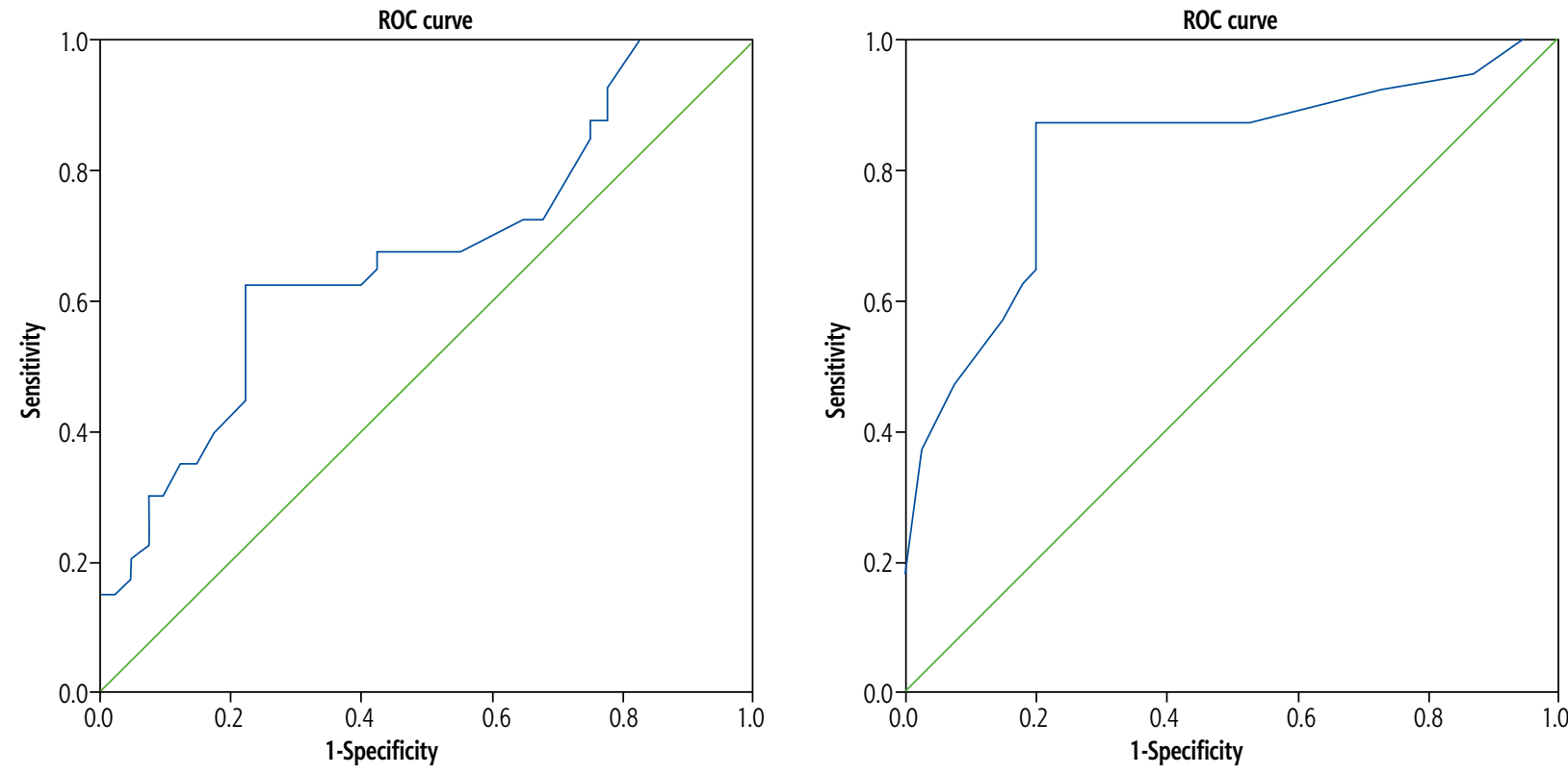

Diagonal segments are produced by ties.

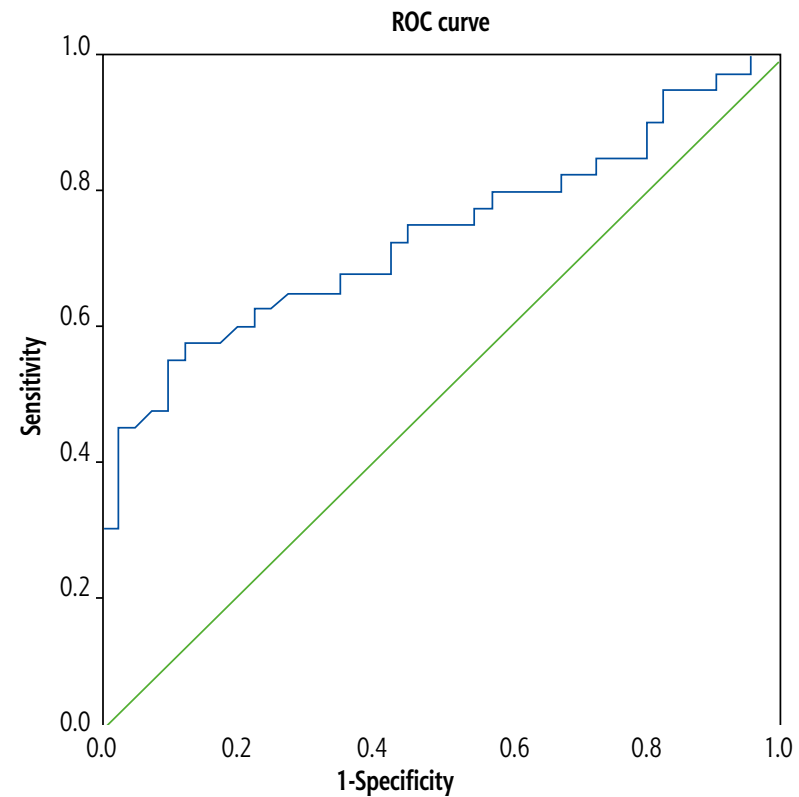

Diagonal segments are produced by ties.

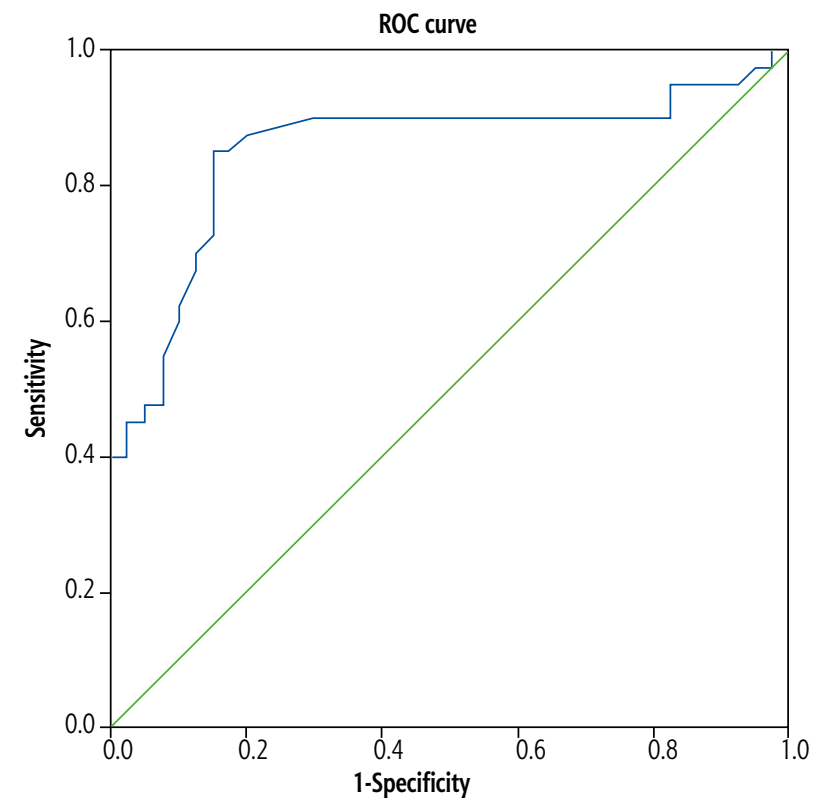

Diagonal segments are produced by ties.

Diagonal segments are produced by ties.

Fig. 1. A) Receiver operating characteristic (ROC) curve demonstrating the accuracy of critical flicker frequency test in the diagnosis of minimal hepatic encephalopathy (MHE) in the study population. B) ROC curve demonstrating the accuracy of modified inhibitory control test in the diagnosis of MHE in the study population. C) ROC curve demonstrating the accuracy of plasma ammonia levels in the diagnosis of MHE in the study population. D) ROC curve demonstrating the accuracy of serum 3-nitrotyrosine levels in the diagnosis of MHE in the study population

Table 5. Accuracy of the optimal cutoff points of different measures and tests of minimal hepatic encephalopathy in the study population

\begin{tabular}{|c|c|c|c|c|c|c|}
\hline Parameter & Cutoff point & Sensitivity (\%) & Specificity (\%) & PPV (\%) & NPV (\%) & Overall accuracy (\%) \\
\hline $\mathrm{CFF}(\mathrm{Hz})$ & $<39$ & 57.5 & 77.5 & 71.9 & 64.6 & 67.5 \\
\hline Modified ICT (unweighted lures) & $>5$ & 87.5 & 80 & 81.4 & 86.5 & 83.8 \\
\hline Plasma ammonia $(\mu \mathrm{mol} / \mathrm{l})$ & $\geqslant 76.45$ & 65 & 72.5 & 70.3 & 67.4 & 68.8 \\
\hline Serum 3-nitrotyrosine $(\mathrm{nmol} / \mathrm{l})$ & $\geqslant 14.15$ & 85 & 82.5 & 82.9 & 84.6 & 83.8 \\
\hline
\end{tabular}

CFF - critical flicker frequency, ICT - inhibitory control test, NPV - negative predictive value, PPV - positive predictive value 
Table 6. Multivariate analysis of predictors of minimal hepatic encephalopathy in the study population

\begin{tabular}{|c|c|c|c|}
\hline Parameter & OR & $95 \% \mathrm{Cl}$ & $p$-value \\
\hline Age (years) & 1.07 & $0.93-1.24$ & 0.357 \\
\hline FIB-4 index & 2.12 & $0.85-5.29$ & 0.108 \\
\hline Child-Pugh score & 0.43 & $0.03-7.18$ & 0.556 \\
\hline \multicolumn{4}{|l|}{ Critical flicker frequency test } \\
\hline$\geqslant 39 \mathrm{~Hz}$ & Reference & Reference & \multirow[t]{2}{*}{$0.04^{*}$} \\
\hline$<39 \mathrm{~Hz}$ & 10.2 & $1.11-93.79$ & \\
\hline \multicolumn{4}{|c|}{ Modified ICT (unweighted lures) } \\
\hline$\leqslant 5$ & Reference & Reference & \multirow[t]{2}{*}{$0.002^{*}$} \\
\hline$>5$ & 43.23 & 3.9-479.59 & \\
\hline Plasma ammonia $(\mu \mathrm{mol} / \mathrm{l})$ & 1.02 & $0.99-1.05$ & 0.153 \\
\hline \multicolumn{4}{|l|}{ Serum 3-nitrotyrosine } \\
\hline$<14.15 \mathrm{nmol} / \mathrm{l}$ & Reference & Reference & \multirow[t]{2}{*}{$<0.001 *$} \\
\hline$\geqslant 14.15 \mathrm{nmol} / \mathrm{l}$ & 50.4 & $5.72-444.16$ & \\
\hline
\end{tabular}

Cl - confidence interval, ICT - inhibitory control test, OR - odds ratio, *significant p-value

levels $\geq 14.15 \mathrm{nmol} / \mathrm{l}(\mathrm{OR}=50.4, p<0.001)$ were significant independent predictors of MHE in the study population after adjusting for age, FIB-4 index, ChildPugh score and plasma ammonia levels (Table 6).

\section{Discussion}

The prevalence of MHE in patients with liver cirrhosis can be as high as $80 \%$ [6]. It has also been linked to poor driving outcomes in addition to affecting other domains of the patient's life, particularly causing a poor health-related quality of life, an impact on socioeconomic status and a higher incidence of falls $[20,21]$. Early diagnosis of MHE is therefore crucial for enhanced quality of life for patients with liver cirrhosis, and for the safety of the patient and the public. While psychometric tests remain the gold standard for MHE diagnosis [10], they are still costly and time-consuming. As a result, alternative diagnostic tests have emerged $[12-14,16]$. However, the latter have not been adequately validated.

We found that, compared to the conventional PHES, a modified version of ICT and serum 3-nitrotyrosine levels possessed the highest accuracy in diagnosing MHE in patients with compensated liver cirrhosis. At cutoff points of $>5$ unweighted lures and $14.15 \mathrm{nmol} / \mathrm{l}$, the modified ICT and serum 3-nitrotyrosine levels, respectively, had an overall accuracy of $83.8 \%$ each in diagnosing MHE in the study population. Additionally, the modified ICT and serum 3-nitrotyrosine levels had the largest AUROCs of $0.827(p<0.001)$ and 0.854 $(p<0.001)$, respectively, in the diagnosis of MHE.
The ICT is a computerized test of attention and response inhibition that has been used to characterize attention deficit disorder, schizophrenia, and traumatic brain injury [22-26]. In our study, we utilized a modified version of the test that uses the numbers $7 / 8$ in Arabic $(v / \wedge)$ as targets in lieu of the traditional $\mathrm{X} / \mathrm{Y}$ - a modification to fit the Egyptian patients better. Patients in the control group scored significantly lower unweighted lures on the modified ICT, compared to the MHE group $(4.9 \pm 2.47$ vs. $8.65 \pm 3.24, p<0.001)$.

In the current work, the modified ICT had an AUROC of 0.827. At a cutoff point of $>5$ unweighted lures, the modified ICT had the highest accuracy in diagnosing MHE in the study population (83.8\%). Among the published studies, the optimal lure cutoff had been notably variable. In a study by Bajaj et al. [14], ICT had an AUROC of 0.902 and overall accuracy in the diagnosis of MHE of $82 \%$ at > 5 lures cutoff, which support our findings. Another study by Bajaj $e t$ al. confirmed these findings; at a cutoff of $>5$ lures, the overall accuracy was $90 \%$ with an AUROC of 0.958 [27]. Other studies reported higher optimal cutoffs for ICT in the diagnosis of MHE (14-27.8 lures), although with variable AUROCs (0.7-0.86) and overall accuracy (71.8-85.6\%) [19, 28, 29].

Serum 3-nitrotyrosine has performed remarkably well in our study in diagnosing MHE with an AUROC of 0.854 and an overall accuracy of $83.8 \%$ at a cutoff of $14.15 \mathrm{nmol} / \mathrm{l}$. Montoliu et al. evaluated the utility of different serum biomarkers in the diagnosis of MHE including serum 3-nitrotyrosine levels in two cohorts [16]. In their first cohort, serum 3-nitrotyrosine levels had an AUROC of 0.96 with an overall accuracy of 
$91 \%$ at a cutoff of $14 \mathrm{nmol} / \mathrm{l}$. When applying the same cutoff in their second cohort of patients, the accuracy of 3-nitrotyrosine levels was $88.5 \%$.

Our study had several limitations. Firstly, the study was performed at a single center; despite being a tertiary referral center, it limited the potential for generalization of the findings without validation in future multicenter clinical trials. Secondly, the limited sample size in our study - despite the difficulties in performing four modalities for the same patient at the same session - might be considered a weakness. However, being a study from Egypt with the highest rates of HCV with its consequences along with the highest rates of car accident mortalities and morbidities might have added substantial value to this study. Additionally, the authors thought that it might be the first study on this topic which assembled and evaluated four diagnostic modalities for the same groups of patients.

Collectively, early detection of MHE in cirrhotic patients has become a necessity in clinical practice. Identification of patients with MHE will allow their treatment, therefore improving their quality of life.

Conclusively, serum level of 3-nitrotyrosine - as a biochemical marker - has proved the highest efficacy in identifying the presence of MHE in mostly illiterate Egyptian patients with liver cirrhosis. ICT is also sensitive, reliable, valid, inexpensive, and can be administered by clinic personnel without the need for psychologic expertise, and can be relied on for MHE detection. Both former tests are suitable for the Egyptian population with special educational and medical situation. CFF was the least and last preferable modality in this study, while ammonia had poorly performed in MHE detection due to other interfering factors regarding the clinical status of the patients.

\section{Ethical statement}

The study complied with the ethical standards of research underpinned by five core values of ethical research, consistent with the National Statement on Ethical Conduct in Human Research: informed consent, voluntary participation, privacy and confidentiality, justice and beneficence, and right to review.

\section{Disclosure}

The authors declare no conflict of interest.

\section{References}

1. American Association for the Study of Liver Disease and European Association for the Study of the Liver. Hepatic encephalopathy in chronic liver disease: 2014 practice guideline by the
European Association for the Study of the Liver and the American Association for the Study of Liver Diseases. J Hepatol 2014; 61: 642-659.

2. Tapper EB, Finkelstein D, Mittleman MA, et al. A quality improvement initiative reduces 30-day rate of readmission for patients with cirrhosis. Clin Gastroenterol Hepatol 2016; 14: 753 759 .

3. Tapper EB, Halbert B and Mellinger J. Rates of and reasons for hospital readmissions in patients with cirrhosis: a multistate population-based cohort study. Clin Gastroenterol Hepatol 2016; 14: 1181-1188.e2.

4. Bismuth M, Funakoshi N, Cadranel JF, et al. Hepatic encephalopathy: from pathophysiology to therapeutic management. Eur J Gastroenterol Hepatol 2011; 23: 8-22.

5. Parekh PJ, Balart LA. Ammonia and its role in the pathogenesis of hepatic encephalopathy. Clin Liver Dis 2015; 19: 529-537.

6. Bajaj JS. Minimal hepatic encephalopathy matters in daily life. World J Gastroenterol 2008; 14: 3609-3615.

7. Bass NM, Mullen KD, Sanyal A, et al. Rifaximin treatment in hepatic encephalopathy. N Engl J Med 2010; 362: 1071-1081.

8. Seyan AS, Hughes RD, Shawcross DL. Changing face of hepatic encephalopathy: role of inflammation and oxidative stress. World J Gastroenterol 2010; 16: 3347-3357.

9. Ferenci P, Lockwood A, Mullen K, et al. Hepatic encephalopathy - definition, nomenclature, diagnosis, and quantification: final report of the working party at the 11th World Congresses of Gastroenterology, Vienna, 1998. Hepatology 2002; 35: 716721.

10. Weissenborn K, Ennen JC, Schomerus H, et al. Neuropsychological characterization of hepatic encephalopathy. J Hepatol 2001; 34: 768-773.

11. Bajaj JS, Etemadian A, Hafeezullah M, et al. Testing for minimal hepatic encephalopathy in the United States: An AASLD survey. Hepatology 2007; 45: 833-834.

12. Kircheis G, Wettstein M, Timmermann L, et al. Critical flicker frequency for quantification of low-grade hepatic encephalopathy. Hepatology 2002; 35: 357-366.

13. Romero-Gomez M, Cordoba J, Jover R, et al. Value of the critical flicker frequency in patients with minimal hepatic encephalopathy. Hepatology 2007; 45: 879-885.

14. Bajaj JS, Hafeezullah M, Franco J, et al. Inhibitory control test for the diagnosis of minimal hepatic encephalopathy. Gastroenterology 2008; 135: 1591-1600.e1.

15. Bajaj JS, Thacker LR, Heuman DM, et al. The Stroop smartphone application is a short and valid method to screen for minimal hepatic encephalopathy. Hepatology 2013; 58: 11221132.

16. Montoliu C, Cauli O, Urios A, et al. 3-nitro-tyrosine as a peripheral biomarker of minimal hepatic encephalopathy in patients with liver cirrhosis. Am J Gastroenterol 2011; 106: 1629-1637.

17. Amodio P, Ridola L, Schiff S, et al. Improving the inhibitory control task to detect minimal hepatic encephalopathy. Gastroenterology 2010; 139: 510-518, 518.e1-2.

18. Campagna F, Montagnese S, Ridola L, et al. The animal naming test: An easy tool for the assessment of hepatic encephalopathy. Hepatology 2017; 66: 198-208.

19. Sharma P, Kumar A, Singh S, et al. Inhibitory control test, critical flicker frequency, and psychometric tests in the diagnosis of minimal hepatic encephalopathy in cirrhosis. Saudi J Gastroenterol 2013; 19: 40-44.

20. Bajaj JS, Riggio O, Allampati S, et al. Cognitive dysfunction is associated with poor socioeconomic status in patients with cir- 
rhosis: an international multicenter study. Clin Gastroenterol Hepatol 2013; 11: 1511-1516.

21. Roman E, Cordoba J, Torrens M, et al. Falls and cognitive dysfunction impair health-related quality of life in patients with cirrhosis. Eur J Gastroenterol Hepatol 2013; 25: 77-84.

22. Epstein JN, Johnson DE, Varia IM, et al. Neuropsychological assessment of response inhibition in adults with ADHD. J Clin Exp Neuropsychol 2001; 23: 362-371.

23. Ford JM, Gray M, Whitfield SL, et al. Acquiring and inhibiting prepotent responses in schizophrenia: event-related brain potentials and functional magnetic resonance imaging. Arch Gen Psychiatry 2004; 61: 119-129.

24. Garavan H, Ross TJ and Stein EA. Right hemispheric dominance of inhibitory control: an event-related functional MRI study. Proc Natl Acad Sci U S A 1999; 96: 8301-8306.

25. Konrad K, Gauggel S, Manz A, et al. Inhibitory control in children with traumatic brain injury (TBI) and children with attention deficit/hyperactivity disorder (ADHD). Brain Inj 2000; 14: 859-875.

26. Pliszka SR, Liotti M, Woldorff MG. Inhibitory control in children with attention-deficit/hyperactivity disorder: event-related potentials identify the processing component and timing of an impaired right-frontal response-inhibition mechanism. Biol Psychiatry 2000; 48: 238-246.

27. Bajaj JS, Saeian K, Verber MD, et al. Inhibitory control test is a simple method to diagnose minimal hepatic encephalopathy and predict development of overt hepatic encephalopathy. Am J Gastroenterol 2007; 102: 754-760.

28. Taneja S, Dhiman RK, Khatri A, et al. Inhibitory control test for the detection of minimal hepatic encephalopathy in patients with cirrhosis of liver. J Clin Exp Hepatol 2012; 2: 306-314.

29. Gupta D, Ingle M, Shah K, et al. Prospective comparative study of inhibitory control test and psychometric hepatic encephalopathy score for diagnosis and prognosis of minimal hepatic encephalopathy in cirrhotic patients in the Indian subcontinent. J Dig Dis 2015; 16: 400-407. 